\title{
Formación en el trabajo y movilidad laboral ${ }^{1}$
}

\author{
Isabel G arcía Espejo
}

Universidad de 0 viedo. D epartamento de Economía Aplicada. Área de Sociología

igarcia@econo.uniovi.es

\section{Resumen}

El objetivo de esteartículo es analizar la influencia de la formación en el trabajo en el logro de la estabilidad laboral y el progreso profesional. El trabajo se inicia con la exposición de los diferentes enfoques teóricos que relacionan este tipo de formación con la movilidad laboral. Asi mismo, se exponen las características de los puestos de trabajo y de los trabaja dores que desde un punto de vista teórico se asocian con una mayor formación. El análisis empírico se basa en observaciones obtenidas por medio de una encuesta longitudinal que reconstruye la historia laboral de una muestra de jóvenes. Por medio de técnicas de regresión logística y de modelos de Cox de riesgos proporcionales, se analiza la influencia de la formación en el trabajo en el riesgo de ir al paro, en los cambios de empleo que obedecen a la decisión del propio trabajador y en el progreso profesional dentro de la empre sa. Los resultados indican que el logro de la estabilidad laboral y la consolidación de una carrera profesional dependen más del acceso a un aprendizaje específico en los primeros empleos que de la acumulación de experiencia laboral o de credenciales educativas.

Palabras clave: cualificaciones profesionales, inserción laboral, estabilidad laboral.

\section{Abstract}

The purpose of this article is to analyze the effects of on-the job training on the attainment of job stability and professional advancement. The paper begins with an introduction to the different theoretical approaches relating this type of training with career mobility. In addition, the job and worker characteristics theoretically associated with a higher educational level are presented. The empirical analysis is based on observations obtained through a longitudinal survey which reconstructs the work histories of a sample of young people. By means of logistic regression techniques and Cox proportional hazard models, the effects of on-the-job training on the following is analyzed: the risk of becoming unemployed, voluntary job changes and professional advancement within companies. The results indicate that the attainment of job stability and the establishment of a pro-

1. Este artículo se integra en una investigación financiada por la CICYT, dentro del Programa $\mathrm{N}$ acional de Estudios E conómicos, Sociales y Culturales, denominada «Procesos de inserción laboral: estrategias, itinerarios y contextos locales» (Proyecto PBS91-1067), dirigida por Rodolfo Gutiérrez y vinculada a una línea de estudios sobre empleo juvenil y tránsito a la vida activa que se viene desarrollando en los últimos años en el Área de Sociología de la Universidad de 0 viedo. 
fessional career depend more on access to specific training in initial jobs than on the mere accumulation of work experience or of educational credentials.

Key words: professional qualifications, transition to job, job stability.

Sumario

Introducción La formación en el trabajo

Enfoques teóricos sobre la relación

Conclusiones

entre formación en el trabajo

y movilidad laboral

Anexo: Definición de variables

Bibliografía

\section{Introducción}

Este artículo trata de la importancia que tiene el acceso a la formación en el trabajo para el logro de la estabilidad y el progreso laboral. La falta de expe riencia laboral o de cual ificaciones adquiridas en la empresa se ha considerado un obstáculo importante para aquellos jóvenes que acceden por primera vez al mercado de trabajo. Como prueba de las dificultados de inserción que presentan estos jóvenes, basta observar la propia duración del paro, significativamente mayor que la duración media de los desempleados que ya tienen experiencia laboral (Alba-Ramírez, 1996; Pérez Infante, 1988). Precisamente, gran parte de las políticas públicas de fomento del empleo que comienzan a establecerse en nuestro país a partir de 1984 han estado orientadas a facilitar a los jóvenes un aprendizaje laboral que les permita adquirir las cualificaciones que no se aprenden en su totalidad en la escuela, a la vez que un conocimiento del mundo del trabajo (disciplina, espíritu de cooperación, etc.) que puede ser valorado por el empresario.

En relación a tales políticas, si bien se puede afirmar que han conseguido reducir de forma notableel número de parados que buscan su primer empleo, también han contribuido a que el proceso de inserción laboral en los últimos años se caracterice por la entrada a la ocupación a través de puestos de trabajo no cualificados, y por una excesiva rotación por este tipo de empleos (García Blanco y G utiérrez Palacios, 1996). Una inserción con tales características ha supuesto en muchos casos que la experiencia laboral lograda no lleve consigo una adquisición de cualificaciones profesionales que puedan ser transferibles a otros empleos, o bien una inversión en formación que haga que resulte gravosa para la empresa la pérdida del trabajador. Esto nos lleva a plantear que quizá no toda experiencia de trabajo tiene efectos positivos de cara a la estabilización y a la carrera laboral, sino sólo aquella que lleva consigo una formación que suponga un incremento significativo del capital humano. 
A este respecto, existen considerables aportaciones teóricas que relacionan la formación en el trabajo con el logro de la estabilidad laboral y con el desarrollo de una carrera profesional, si bien la investigación empírica es bastante menor, sobre todo en el ámbito español. A partir de estas consideraciones, en este artículo se analiza precisamente cómo influye la formación adquirida en el trabajo en la movilidad laboral en un sentido amplio, considerando su influencia en el riesgo de ir al paro, en los cambios de empleo que obedecen a la decisión del propio trabajador y en el progreso laboral dentro de la empresa.

Enfoques teóricos sobre la relación entre formación en el trabajo y movilidad laboral

La importancia de la formación en el trabajo como generadora de cualificaciones profesionales ha sido señal ada en numerosas ocasiones y desde diversas perspectivas teóricas. Esta idea está especialmente presente en una serie de teorías que podríamos denominar credencialistas. D entro de esta perspectiva, autores como Arrow (1991), Spence (1991) y Thurow (1975) consideran que la formación en el trabajo es la que verdaderamente genera las cualificaciones que el trabajador va a necesitar para desarrollar su tarea, mientras que la educación formal actuaría más bien como un mecanismo de selección que informa al empresario de la capacidad del individuo para ser formado. Tal concepción no niega que exista una cierta acumulación de capital humano durante el período de educación formal, si bien se considera que es en el período poseducacional, una vez que el individuo accede al empleo y acumula experiencia laboral, cuando se adquieren las cualificaciones que suponen un incremento significativo del capital humano.

Similar argumentación se encuentra en la tradición de la segmentación de los mercados de trabajo, para la cual las cualificaciones se adquieren también básicamente en el trabajo. La educación formal se usa más como un recurso de selección para buscar trabajadores que posean determinadas actitudes y rasgos de comportamiento. En la misma dirección que las teorías credencialistas, la educación sería sobre todo un mecanismo de selección; la formación en el trabajo es la que proporciona la mayor parte de las cualificaciones utilizadas en la realización de las tareas del puesto (D oeringer y Piore, 1985).

El propio modelo teórico del capital humano se inicia con el análisis de la formación en el trabajo antes de abordar la educación formal. Para este modelo, una vez que finaliza la educación formal y el individuo accede a la ocupación, la inversión en capital humano se realiza mediante la formación en el puesto. Becker (1983) distingue dos tipos de formación: general y específica. La formación general es aquélla que resulta útil no sólo para la empresa que la proporciona, sino también para el resto de empresas. Este tipo de formación no cualifica únicamente para ocupar un puesto específico en una determinada empresa y puede utilizarse posteriormente en cualquier otro empleo. Según su formulación más sencilla, los empresarios no pagan la formación 
general porque el trabajador no tiene ningún incentivo para permanecer en la empresa, dado que puede obtener un mayor salario en cualquier otra. Es por ello que las empresas suelen proporcionar formación general repercutiendo los costes a los trabajadores, los cuales reciben un salario menor a su productividad durante el período formativo.

Por su parte, la formación específica es aquélla que aumenta la productividad del individuo en mayor medida en las empresas que la proporcionan. Según esta idea, el salario que obtendría un trabajador en otra empresa es independiente de la formación que ha recibido y, por tanto, las empresas son las encargadas de hacer frente a los costes de este tipo de formación, puesto que ningún empleado estaría interesado en asumir los costes de una formación de dudoso beneficio.

Sin embargo, en la práctica no resulta tan fácil distinguir entre formación general y específica, o entre cualificaciones generales y específicas. Tampoco hay suficiente evidencia empírica de que los trabajadores paguen efectivamente sus cualificaciones generales percibiendo un menor salario durante su período formativo. A este respecto, algunas investigaciones han encontrado que los trabajadores de las empresas que suministran una mayor cantidad de formación, o que reciben una mayor formación, perciben unos salarios más al tos que los trabajadores que no adquieren formación (Barron, Black y Loewenstein, 1989). Tales evidencias parecen sugerir dos cuestiones: por un lado, que ambos tipos de formación son más bien complementarios y difíciles de distinguir; por otro, que no es tanto la naturaleza de la formación lo que determina las decisiones de financiación por parte del empresario, sino más bien la probabilidad de pérdida de la inversión que ha efectuado en formación si el trabajador abandona la empresa.

La asociación entre formación en la empresa y rotación laboral constituye, precisamente, un aspecto en el que existe una gran coincidencia teórica. Tanto la perspectiva del capital humano, como las teorías de la segmentación o la corriente teórica de los mercados internos de trabajo señalan como la rotación resulta importante cuando existen costes de formación que tienen que soportar los trabajadores o las empresas. Si una empresa paga la formación de un trabajador y éste se marcha, la inversión efectuada por la empresa se pierde. Si, por el contrario, un trabajador es despedido después de haber invertido en formación (fundamentalmente específica), sufrirá una pérdida de capital. Es por ello que las empresas tratarán de evitar la rotación laboral a través de diversos mecanismos.

U no de los procedimiemtos para evitar la rotación laboral es el establecimiento de mercados internos de trabajo. A este respecto, la formación específica ha sido considerada como un factor muy relacionado con la generación de los mercados internos (K noke y Kalleberg, 1994). D oeringer y Piore (1985) consideran a la formación específica como el elemento fundamental para el surgimiento de estos mercados. Estetipo de formación es la que hace que la rotación sea costosa para la empresa porque el empresario ha tenido que invertir en ella. El empleador tendrá necesidad de establecer ciertas políticas para desa- 
lentar la rotación de la mano de obra. Son estas políticas que tratan de reducir la rotación, así como minimizar los costes de la formación específica, las que engendran mercados de trabajo internos. Estos mercados disminuyen las pre siones competitivas de los mercados de trabajo sobre empleadores y asalariados, y se caracterizan por la existencia de relaciones de empleo a largo plazo y el establecimiento de carreras de promoción internas que constituyen el proce dimiento básico que regula la asignación de posiciones y salarios. Su creación resultaría una medida eficaz, tanto para los empleadores como para los trabajadores (D oeringer y Piore, 1985). El establecimiento de mercados internos asegura la colaboración de los trabajadores con experiencia en la formación de los nuevos contratados, al imponer un cierto grado de rigidez salarial y de seguridad en el empleo.

La importancia de asegurar la formación por parte de los trabajadores veteranos está también presente en otros model os teóricos como el modelo de competencia por las vacantes (Sorensen y Tuma, 1981; Sorensen y Kalleberg, 1981), que establece una segmentación del mercado de trabajo en torno a la diferenciación entre relaciones de empleo abiertas o cerradas. La formación en el trabajo tiene una gran importancia en el surgimiento de relaciones de empleo cerradas, aquéllas en las que el empleador no tiene el control sobre el acceso al trabajo; fundamentalmente la formación específica. Para que la formación en el trabajo sea efectiva, es necesario contar con la cooperación de los trabajadores veteranos, y esta cooperación puede no producirse si la competencia por los salarios prevalece dentro de la empresa. Reconocer a los empleados mayor control sobre el trabajo es una forma que tienen los empresarios de asegurar que la formación tiene lugar de hecho.

Tampoco las cualificaciones específicas han sido el único tipo de cualificaciones señaladas como generadoras de mercados internos, con el objetivo de evitar la rotación laboral. M arsden (1994) señala que las cualificaciones transferibles, que son características de los mercados profesionales, también dan lugar al surgimiento de mercados internos como forma de proteger la inversión que la empresa ha efectuado en formación. Si la cualificación es transferible, cabría pensar, en principio, que los costes deberían ser soportados por los trabajadores por medio de mecanismos tales como un salario menor durante la etapa de aprendizaje. N o obstante, M arsden señala que existen diferentes razones para que las empresas soporten la totalidad o parte de la formación. Una de ellas es la resistencia por parte de los trabajadores veteranos a aceptar que los aprendices perciban un salario menor por miedo a ser sustituidos por ellos. Una solución a esta resistencia puede ser la financiación del Estado a través de la formación profesional, pero aun así esta formación no proporciona la experiencia en el trabajo que sólo se puede adquirir en las empresas y que es costosa. $M$ arsden concluye que a falta de medios eficaces para repartir los costes de formación, las empresas tenderán a desarrollar más sus propios mercados internos como medio de proteger su inversión.

Puesto que la formación en el trabajo constituye un factor muy importante de cara al logro de la estabilidad laboral, resulta especial mente rel evan- 
te determinar qué características de los puestos y de las personas se asocian a una mayor formación. A este respecto, diversas investigaciones han señalado ciertas características de los empleos y de los trabajadores que favorecen su adquisición.

El tamaño de la empresa ha sido un factor relacionado con la formación en el empleo. Parece que las mayores empresas invierten más en la formación de los trabajadores. Varias son las razones citadas para explicar este comportamiento. Se habla de que tales empresas pueden beneficiarse de economías de escala en la provisión de la formación, de que tienen mayor capacidad para efectuar una selección más rigurosa e intensiva, sufren una rotación laboral menor y pueden efectuar una asignación más óptima de su mano de obra (Knight y Latreille, 1996; G reenhalgh y M avrotas, 1994; Green, 1993).

Además del tamaño de la empresa, la formación en el trabajo parece ser más intensa en industrias expansivas, de rápido crecimiento 0 en industrias con grandes cambios tecnológicos, así como en las categorías ocupacionales que están más sujetas a cambios, principalmente en ocupaciones de al to nivel (G reen, 1993). D e igual forma, como ya se ha expuesto anteriormente, la presencia de mercados internos en las empresas constituye un elemento estrechamente relacionado con la formación en el trabajo.

Si nos referimos a las características de los trabajadores, son frecuentes las investigaciones que señalan un acceso diferencial a la formación entre hombres y mujeres (G reenhalgh y Stewart, 1987; Lynch, 1992; Booth, 1991). Las razones de este acceso diferencial se han buscado en la interacción de factores de la oferta y la demanda de trabajo. D esde el lado de la oferta se habla de las diferencias en la propia elección de estudios que hace que las chicas se alejen de aquellas ocupaciones dominadas por los hombres y que típicamente requieren formación técnica (Allison y Allen, 1980; Filer, 1986), y de las diferencias de expectativas sobre la participación laboral en el futuro que determina el tipo y cantidad de capital humano invertido (Sandell y Shapiro, 1980). De parte de la demanda, la menor formación en el trabajo que reciben las mujeres en relación con los varones se ha imputado, desde la perspectiva del capital humano, al hecho de que las mujeres tiene una mayor probabilidad de abandono del trabajo, generalmente por motivos familiares. Este comportamiento daría lugar a una pérdida de la inversión en formación efectuada por las empresas y, por ello, las empresas suelen destinar la formación a los trabajadores más estables, en este caso a los varones. Pero ésta no es la única explicación posible. Así, desde una perspectiva estructural, las diferencias de formación entre hombres y mujeres se deberían fundamentalmente al tipo de empleos destinados a uno u otro sexo, es decir, a la segregación ocupacional (G reen, 1993; Booth, 1993; I báñez Pascual, 1999).

Respecto a otras características personales como la educación, las investigaciones realizadas ofrecen resultados contradictorios. M ientras al gunas de ellas señalan que los trabajadores de mayor nivel educativo ocupan los empleos que requieren un mayor período de formación (Altonji y Spletezer, 1991), otras investigaciones no encuentran relación o ésta es muy débil, especialmente 
cuando se trata de formación adquirida en el propio puesto de trabajo (Lynch, 1992).

Por último, la presencia de sindicatosy el propio marco institucional en el que se configuran las relaciones de empleo (derechos de antigüedad, de pensión, sistemas de remuneración, legislación contractual, etc.) constituyen factores a tener en cuenta a la hora de analizar la formación en el trabajo (Greenhalgh y M avrotas, 1994). Este marco institucional puede influir en la forma predominante de organización de la formación. Así, por ejemplo, en algunos países la pauta dominante de formación puede consistir en el aprendizaje de un oficio seguido de una alta movilidad entre empresas, mientras que en otros dicha formación puede estar más orientada hacia los mercados internos de trabajo (OCDE, 1994).

\section{La formación en el trabajo}

A partir de estas consideraciones teóricas, en este artículo se persiguen varias cuestiones. En primer lugar, determinar qué características de las empresas se hallan más asociadas con la formación en el trabajo y qué atributos personales son recompensados con una mayor formación. En segundo lugar, analizar su influencia sobre la movilidad inicial, una vez que se accede al primer empleo, considerando, por un lado, la movilidad que va de la ocupación al paro, para ver en qué medida la formación reduce la rotación laboral de tipo involuntario y, por otro, los cambios entre empleos que obedecen a la decisión del propio trabajador y que podrían considerarse como una rotación laboral de tipo voluntario. Asimismo, se analiza la movilidad interna o promocional.

El análisis empírico se ha basado en la información obtenida por medio de una encuesta de carácter longitudinal realizada a 2.000 jóvenes ${ }^{2}$ con edades comprendidas entre 20 y 29 años residentes en el área metropolitana de Asturias. En esta encuesta se reconstruye la historia laboral de los jóvenes desde su entrada al mercado de trabajo hasta el momento de la encuesta. Por medio de técnicas de regresión logística y de riesgos proporcionales se han construido mode los en los cuales la variable que mide la formación en el trabajo se va a poner

2. El error muestral es del $\pm 2,2 \%$, con un nivel de confianza del $95,5 \%$. La encuesta se realizó en junio de 1992 a una muestra de jóvenes referida al universo de población de 20 a 29 años residente en el llamado ocho asturiano, integrado por las áreas urbano-industriales de la costa (Avilés y Gijón, junto con los municipios de su área de influencia Cor vera y Castrillón), del centro urbano (0 viedo y Siero) y de las zonas de tradición minera del interior (Langreo y M ieres). La muestra se ha seleccionado por un procedimiento polietápico. U na vez elegidos los municipios y estratificada la muestra, se seleccionaron las secciones censales de forma al eatoria simple. La elección de los individuos a entrevistar se efectuó por medio del procedimiento de rutas aleatorias con cuotas de sexo, edad y experiencia laboral. El motivo de establecer esta última cuota obedeció al deseo de asegurar la correcta representación de los estudiantes, que podrían estar sobrerrepresentados al tener mayores probabilidades deencontrarse en el hogar en el momento de la encuesta. U na información más detallada de la encuesta puede verse en García Espejo (1998). 
en relación con otras variables que pueden influir también en la movilidad laboral ${ }^{3}$.

Las primeras indagaciones analíticas han estado dirigidas a conocer las características personales y aquéllas que están relacionadas con los puestos que se asocian con la formación en el trabajo. Como medida de la formación se ha utilizado una pregunta del cuestionario en la que se indaga sobre el tiempo de aprendizaje que el trabajador considera necesario para desempeñar correctamente su trabajo. Las posibles respuestas son: 1. «N inguno», 2. «M enos de 1 mes», 3. «D e 1 a 3 meses», 4. «D e 3 a 12 meses», 5. «M ás de 1 año». Esta medida dela formación en el trabajo esfrecuente en las investigaciones empíricas rea lizadas al efecto 4 .

La población de referencia está constituida por los trabajadores asalariados. Según los resultados obtenidos referidos al primer empleo, el $47 \%$ de los trabajadores declaran necesitar menos de un mes de aprendizaje, un $24 \%$ de uno a tres meses y el $29 \%$ restante más de tres meses (tabla 1 ). El elevado porcentaje de trabajadores que declaran no necesitar ningún tiempo de formación no resulta excesivamente sorprendente, si tenemos en cuenta que se está analizando el primer empleo de los jóvenes. En España, gran parte de la generación de empleo juvenil se ha efectuado a través de trabajos no cualificados que requieren muy poca formación.

Para determinar, precisamente, qué características personales se encuentran más asociadas a la formación en el trabajo y qué características de los puestos y de las empresas se relacionan con esta formación, se ha efectuado un modelo de regresión logística en el que la variable dependiente es el tiempo de aprendizaje recodificada en dos categorías: 1 «tiempo de aprendizaje superior a tres meses» y 0 «tiempo de aprendizaje inferior a tres meses». La pobla ción de referencia está constituida por los trabajadores asalariados, excluyendo los empleadores, autónomos y ayudas familiares (tabla 2). Las características personales de los individuos están representadas por los recursos formativos, medidos a través de la titulación, las situaciones de simultaneidad de estudios y trabajo, el tiempo de paro previo hasta obtener el empleo y el sexo. Para ana

3. En las tablas donde se presentan los resultados obtenidos, sólo figuran las variables que han resultado ser estadísticamente significativas. En todos los modelos se ha aplicado el método de selección de variables denominado Forward o de incorporación progresiva de las variables independientes, que consiste en ir añadiendo los posibles predictores o variables independientes de una en una, manteniendo en el modelo las que son estadísticamente significativas y descartando las que no lo son. El criterio de significación estadística que se ha utilizado en este artículo es el 95,5\%. Por otro lado, en el uso de variables explicativas categóricas es necesaria la utilización de un término independiente que no aparezca en el modelo. En este caso se ha optado por el contraste de desviación del efecto general, que consiste en la comparación de cada una de las categorías de la variable independiente (excepto la que se ha omitido) con el efecto total.

4. La Encuesta de Condiciones de Vida y T rabajo (ECVT) realizada en España en 1985, y la Encuesta de Estructura, C onciencia y Biografía de C lase (ECBC) realizada en 1991 incorporan un indicador similar. 
Tabla 1. Tiempo de aprendizaje necesario para realizar el trabajo.

\begin{tabular}{lc}
\hline & Porcentaje \\
\cline { 2 - 2 } 1. N inguno & 21,4 \\
2. M enos de 1 mes & 26,1 \\
3. De 1 a 3 meses & 24,1 \\
4. De 3 a 12 meses & 14,4 \\
5. Más de 1 año & 14,0 \\
\hline Total & 100,0 \\
\end{tabular}

lizar la influencia del tipo de puestos de trabajo y de las empresas se han incluido en el modelo la categoría ocupacional, la rama de actividad, el tipo de vínculo laboral y el tamaño de la empresa. A estas variables se han añadido las formas de entrada al empleo, representadas por la realización de pruebas de selección y el canal de acceso. Esta última variable adquiere los valores de «1» si el empleo se obtuvo a través del recurso a terceras personas (capital relacional) y «0» por otras vías.

Si analizamos el comportamiento de las características personales, la valoración del mayor tiempo de aprendizaje proviene de los trabajadores con estudios de formación profesional, sobre todo de las especialidades de industria y construcción. Parece, pues, que estos trabajadores adquieren las cualificaciones profesionales fundamentalmente en el puesto de trabajo y no en el sistema edu cativo. A este respecto, es evidente que hay ocupaciones para cuyo aprendizaje puede ser suficiente las aulas y otras que requieren una formación en el propio puesto. Este último caso sería especial mente aplicable a los puestos de trabajo de tipo manual en los que se utilizan materiales y maquinaria de alto coste, que requieren grandes espacios y cuya simulación en el aula resulta difícil (Garrido, 1995).

El tiempo de aprendizaje es sensiblemente inferior en las mujeres que en los hombres ${ }^{5}$. Este resultado parece indicar la existencia de una discriminación de mercado. Se puede afirmar que existe una discriminación de merca do cuando, a igualdad de características personales, hombres y mujeres tienen desiguales probabilidades de recibir formación, como parece que es nuestro caso. N o obstante, a pesar de que pueda existir una igual dad en los recursos personales entre hombres y mujeres, también se ha argumentado frecuentemente que el mayor acceso a la formación por parte de los primeros puede

5. Según datos de la Encuesta de Condiciones de Vida y Trabajo (ECVT), el tiempo de aprendizaje es inferior también para las mujeres que para los varones (Rodríguez Rodríguez, 1993). 
Tabla 2. M odelo LOGIT. Probabilidad de que el tiempo de aprendizaje necesario para realizar el trabajo sea superior a tres meses.

Variables

Tipo de titulación (Categoría residual: EGB)

1. FP1 Industria y Construcción

$* * *$

2. FP1 Administrativa

$0,5831^{*}$

3. FP1 Servicios

$-1,1251^{*}$

4. FP2 Industria y Construcción

0,5382

5. FP2 Administrativa

$0,8610^{* * * *}$

6. FP2 Servicios

$-0,0837$

7. D iplomaturas de Arte y $\mathrm{H}$ umanidades

$0,6237 *$

0,1627

8. Diplomaturas Científico-T ecnológicas

$-0,4875$

9. Diplomaturas de Ciencias Sociales

$-0,2284$

10. D iplomaturas Biosanitarias

0,6440

11. Licenciaturas de Arte y $\mathrm{H}$ umanidades

$-0,4545$

12. Licenciaturas Científico-T ecnológicas

0,1143

13. Licenciaturas de Ciencias Sociales

$-0,3429$

14. Licenciaturas Biosanitarias

$-1,0368 *$

15. BUP-COU

$-0,0037$

Sexo (Categoría residual: mujer)

$0,5046^{* * *}$

Categoría ocupacional (Categoría residual: obreros no cualificados)

***

1. Profesionales y técnicos

$1,3932 * * *$

2. Administrativos y comerciales

$-0,0507$

3. 0 breros cualificados

$-0,1004$

Tipo de vínculo laboral (Categoría residual: sin contrato)

**

1. Contrato indefinido

$0,3235 * *$

2. Contrato temporal

$-0,1908^{* *}$

Rama de actividad (C ategoría residual: servicios personales)

***

1. M inería, Energía y Q uímica

$-0,0421$

2. M etal

0,4301

3. Resto industria

0,3671

4. Construcción

$0,8374 * * *$

5. Transporte y Comunicaciones

$-0,7067^{* *}$

6. Comercio y H ostelería

$-0,6772 * * *$

7. Administraciones públicas

0,3010

8. Enseñanza y Sanidad

$-0,3062$

9. 0 tros servicios

$-0,2027$

Pruebas de selección (Categoría residual: pasar por una selección)

$* *$

1. Pasar por una oposición

0,1271

2. No

$-0,2901^{* *}$

Constante

$-0,5080 * * *$

№ de casos: 1.211/-2LL: 1189,458

Niveles de significación: ${ }^{*} p<0,1000 * * p<0,0500 * * * p<0,0100$. 
obedecer a diferencias en la motivación y en las actitudes. Las mujeres tendrían unas actitudes y motivaciones hacia el trabajo y la carrera laboral diferentes a los hombres.

Podemos determinar si existen diferencias por género en las actitudes hacia el trabajo, analizando cuáles son los elementos que más atrajeron a los jóvenes en su primer empleo (tabla 3). Como puede observarse, no existen diferencias significativas entre hombres y mujeres. Para ambos el elemento más valorado de un empleo es el salario, seguido por la oportunidad de empezar a trabajar y el contenido de las tareas. Además, si nos referimos a las posi bilidades de formación que ofrece el empleo, si bien la diferencia no es muy grande, las mujeres conceden más importancia a este factor que los varones.

0 tra argumentación bastante extendida que intenta explicar la menor formación en el trabajo que reciben las mujeres es aquélla que se centra un su propensión al abandono voluntario del trabajo por motivos familiares. LoS empresarios destinan los puestos que requieren un mayor tiempo de aprendizaje a los trabajadores cuyo comportamiento presumiblemente resulta más estable, en este caso a los varones. En nuestro caso, tal argumentación se puede analizar observando los motivos aducidos por los jóvenes que perdieron su primer empleo (tabla 4). En ambos sexos, el motivo principal fue la finalización del tiempo de contrato. Por parte de los varones, el abandono voluntario que obedece al hecho de haber encontrado un trabajo mejor es superior que en las mujeres, mientras que éstas han abandonado el empleo por motivos familiares en mayor proporción que sus homólogos masculinos. Ahora bien, este comportamiento está bastante relacionado con el nivel educativo, en el sentido de que prácticamente se circunscribe a las mujeres con menor nivel de estudios. Basta señalar que de las mujeres que han dejado el empleo por motivos fami-

Tabla 3. Atracción por el empleo según sexo.

1. El salario.

2. Las tareas del puesto

3. La seguridad en el empleo

4. El prestigio de la empresa.

5. La confianza en el dueño

6. Las condiciones de trabajo

7. La oportunidad de formarse

8. Las oportunidades de promoción

9. La oportunidad de empezar a trabajar

\begin{tabular}{rr} 
Varón & M ujer \\
\hline 40,5 & 35,3 \\
11,8 & 13,7 \\
4,1 & 3,8 \\
1,3 & 0,9 \\
3,2 & 3,0 \\
3,3 & 5,0 \\
8,4 & 10,5 \\
1,3 & 0,8 \\
24,6 & 24,4 \\
1,7 & 2,6 \\
100,0 & 100,0 \\
$(666)$ & $(761)$
\end{tabular}

10. Otros 
Tabla 4. M otivos de la pérdida del primer empleo según sexo.

1. Se cumplió el tiempo de contrato.

2. Encontró otro empleo mejor

\begin{tabular}{rr} 
Varón & M ujer \\
\hline 41,8 & 46,0 \\
23,0 & 17,2 \\
5,6 & 5,2 \\
15,8 & 15,1 \\
1,2 & 11,6 \\
100,0 & 100,0 \\
$(486)$ & $(424)$
\end{tabular}

liares un $45 \%$ tienen estudios de EG B, no hay ninguna licenciada y sólo tiene lugar en un $2 \%$ de las diplomadas universitarias.

La formación en el trabajo está muy relacionada con las características del puesto al que se accede. Las categorías de más alto estatus ocupacional se hallan asociadas de forma positiva con un mayor tiempo de aprendizaje en el puesto. El aprendizaje también es mayor en los trabajadores que tienen una relación contractual indefinida con la empresa. T al resultado sugiere que las empresas aseguran su formación por medio de la estabilidad en el empleo, ya quees pre visible que los trabajadores con contratos indefinidos (que suelen estar rela cionados con la existencia de mercados internos) tengan un menor aliciente para abandonar el empleo.

L a siguiente característica de las empresas que resulta significativa es la rama de actividad. La mayor formación en el puesto proviene de los trabajadores de la rama de Construcción. Ese resultado puede estar relacionado con el hecho de que los oficios característicos de esta rama prácticamente no cuentan con una formación ocupacional de carácter reglado, siendo la formación en el propio puesto la única vía que posibilita la adquisición de las cualificaciones necesarias para desarrollar el trabajo. Además, se trata de puestos donde la adquisición de habilidades y los conocimientos prácticos necesarios son difíciles de adquirir en el aula.

La formación en el puesto afecta también a los mecanismos de selección y contratación de las empresas. Q uela formación en el puesto de trabajo constituye un factor determinante para el establecimiento de procesos de selección de personal, ha sido señal ado en diversas investigaciones (C ohen y Pfeffer, 1986; Barron y otros, 1985; Barron, Black y Loewenstein, 1989). Es de esperar que las empresas sean más selectivas a la hora de elegir un candidato para un puesto de trabajo que requiere un período amplio de aprendizaje, no sólo con vistas a sel eccionar a individuos capaces de adquirir las cualificaciones necesarias para el desarrollo de las tareas, sino también para evitar así contratar trabajadores con tendencia al abandono voluntario, que supondrían para la empresa la pérdida de la inversión efectuada en formación. En este 
aspecto, los resultados indican que cuanta más formación requiera el puesto de trabajo, más rigurosas son las empresas en la selección de los futuros trabajadores. Así, el acceso al empleo sin que sea necesario realizar pruebas de selección está inversamente relacionado con la cantidad de formación necesaria para desarrollar el trabajo.

\section{Formación en el trabajo y movilidad laboral}

Existe una gran coincidencia teórica respecto a que la formación en el trabajo constituye un factor muy importante de estabilidad laboral. D esde el lado de la demanda de trabajo, los empleadores han invertido en una formación que pierden si el trabajador abandona la empresa y, por tanto, es de esperar que este tipo de formación ejerza un efecto protector frenteal desempleo. D esde el lado de la oferta, Ios trabajadores pueden haber adquirido una formación específica poco transferible a otras empresas distintas de aquélla que proporcionó dicha forma ción, y éste hecho da lugar que los trabajadores tengan menos alicientes para buscar otro trabajo. Consecuentemente, la movilidad entre empleos será menor. Además, los puestos de trabajo que ofrecen mayor formación suelen estar asociados a condiciones laboral es estables, como se deduce de su relación con la contratación indefinida, y éste es otro factor que desincentiva la movilidad.

Q ue la formación en el puesto de trabajo constituye un factor protector frente al paro, a la vez que inhibe la movilidad entre empleos, constituyen los aspectos a considerar en este apartado. Para ello, se ha acudido a la elaboración de modelos de $\mathrm{C}$ ox de riesgos proporcionales, que permiten determinar el riesgo de que ocurra una determinada transición de un estado a otro según una serie de variables independientes. Esta técnica está especial mente indicada para analizar historias de eventos. En la encuesta que sirve de base a este artículo, la trayectoria laboral de los individuos fue diseñada como una historia de eventos. Se puede definir una historia de eventos como un registro acerca de la secuencia temporal de cambios y de los estados discretos (en este caso relacionados con la actividad laboral) ocupados por una muestra de individuos.

Las variables con coeficientes positivos se hallan asociadas a decrementos del intervalo temporal entre dos eventos o sucesos y señalan un mayor riesgo de movilidad, mientras que las variables con coeficientes negativos estarán asociadas a incrementos de dicho intervalo temporal e indican que el riesgo de efectuar una determinada transición será menor ${ }^{6}$.

6. El modelo de regresión de Cox puede ser descrito en términos de funciones de supervivencia:

$$
\mathrm{S}(\mathrm{t})=\left(\mathrm{S}_{0}(\mathrm{t})\right)^{\mathrm{p}}
$$

D onde $S(t)$ representa la probabilidad de supervivencia después del instante $t, S_{0}(t)$ es la Ilamada función línea-base de supervivencia, dependiente exclusivamente del tiempo y similar a la constante en las regresiones múltiples, y p que depende de las covariantes y de sus coeficientes $B$, siendo

$$
p=e^{X B}
$$


Formación en el trabajo y riesgo de desempleo

El estudio de la influencia de la formación en el trabajo y el riesgo de desempleo se ha efectuado mediante la consideración de las transiciones que van de la ocupación al paro. La variable dependiente en el modelo construido al efecto es el número de meses que se permanece en un empleo antes de moverse hacia el paro o de ser censurado. El análisis se refiere al siguiente suceso después de la primera ocupación. Al considerar el primer empleo se elimina el efecto que podría ejercer en la estabilidad laboral y en otros tipos de movilidad la forma ción recibida en empleos anteriores, y que es difícil de determinar. Los mode los recogen el tiempo de aprendizaje junto a una serie de variables explicativas, que según la literatura sobre movilidad laboral influyen tanto en las transiciones entre ocupación y paro como en la movilidad directa entre empleos. Estas variables están representadas por los recursos personales y por las recompensas del trabajo (Sorensen y Tuma, 1981; Tuma, 1985; Blossfeld, 1989).

Como recursos personales, en el modelo se incluye el nivel de estudios del trabajador en el momento de la transición, distinguiendo si está completado o no, y el capital relacional medido por el canal de acceso al empleo. Las recompensas del trabajo están representadas por una variable que mide la obtención o no de un ascenso, por el tipo de vínculo laboral, por la categoría ocupacional y por una variable que mide la fecha de entrada en la ocupación, antes o después de 1984, como un factor de gran importancia en la explicación dela estabilidad laboral. Es en esta fecha cuando se produce un cambio en las normas de contratación en nuestro país, que va a dar lugar a un incre mento notable de la contratación temporal. También se ha considerado la posible influencia de ciertas características del empleo, como la rama de actividad y el tamaño de la empresa. Finalmente, se incluye la variable sexo para recoger las posibles diferencias en las pautas de movilidad entre hombres y

La función de supervivencia se relaciona con la denominada función de riesgo $\mathrm{h}(\mathrm{t})$, que mide el riesgo en un instante determinado; el ratio de fallo por unidad de tiempo. N ormalmente se prefiere trabajar con esta función por ser más sencilla, siendo entonces el model o:

$$
h(t)=\left(h_{0}(t)\right) e^{(B x)}
$$

La función de riesgo, como la función de supervivencia, está factorizada en dos componentes: la línea base de riesgo $h_{0}(t)$ que depende exclusivamente del tiempo, mientras que ${ }^{(\mathrm{BX})}$ depende sólo del valor de las variables independientes y de los coeficientes de regresión.

El modelo de Cox es comúnmente expresado en forma de riesgo y es llamado modelo de riesgos proporcionales Se llama así por el hecho de que para dos casos dados, el ratio de sus riesgos es una constante en el tiempo, siempre que las variables independientes no cambien en el tiempo.

Tomando logaritmos, la forma general del modelo es:

$$
\log h(t)=a(t)+B_{1} x_{1}+B_{2} x_{2}+\ldots+B_{p} X_{p}
$$

$D$ onde $a(t)$ es una función del tiempo. $D$ ado que esta función no tiene que ser especificada, el modelo es descrito como parcialmente paramétrico o semiparamétrico. 
Tabla 5. M odelo Cox. T ransiciones de una situación de ocupación en el primer empleo a una situación de paro.

Tiempo de aprendizaje (Cat. residual: $N$ inguno)

1. M enos de 1 mes

***

2. De 1 a 3 meses

$0,2263^{* *}$

3. De 3 a 12 meses

0,1135

4. M ás de 1 año

$-0,1506$

Ascenso en el trabajo de origen (Cat. residual: $\mathrm{N}$ o)

$-0,3707^{* * *}$

Año de entrada en la ocupación (Cat. residual: Antes de 1984)

$-0,4598 * *$

$0,6861^{* * *}$

Tipo de vínculo laboral (C at. residual: Sin contrato)

1. Contrato indefinido

$-1,3440 * * *$

2. Contrato temporal

$0,9374 * * *$

Rama de actividad (C at. residual: Servicios personales)

$* * *$

1. M inería, Energía y Q uímica

$-0,0106$

2. M etal

3. Resto industria

$0,5899 * * *$

4. Construcción

$-0,2397$

5. T ransporte y Comunicaciones

$0,3215^{*}$

6. Comercio y H ostelería

$-0,2299$

7. Administraciones públicas

$-0,0519$

8. Enseñanza y Sanidad

$0,4208 * *$

9. 0 tros servicios

$-0,3198 *$

$-0,1484$

Tamaño de la empresa (Cat. residual: N o contesta)

***

1. M enos de 10 empleados

0,0709

2. De 10 a 50 empleados

$-0,0703$

3. Más de 50 empleados

Sexo (C at. residual: $M$ ujer)

$-0,2508 *$

$-0,2778 * *$

$\mathrm{N}$ ㅇ de casos: 1.216 / C asos no censurados: 432/-2LL: 5292,832

Niveles de significación: ${ }^{*} p<0,1000 * * p<0,0500 * * p<0,0100$.

mujeres, y el área de residencia como un indicador indirecto de las oportunidades laborales existentes en un momento dado en una zona determinada (tabla 5).

La interpretación de los resultados se centra en el comportamiento de la variable tiempo de aprendizaje. Las variables con coeficientes positivos se hallan asociadas a decrementos del interval o temporal entre dos eventos y señalan un mayor riesgo de desempleo, mientras que las variables con coeficientes negativos están asociadas a incrementos de dicho intervalo temporal e indican que el riesgo de efectuar una transición de la ocupación al paro será menor. 
Tal como muestran los resultados obtenidos, el tiempo de aprendizaje sí constituye un factor que contribuye al logro de la estabilidad laboral, dado que cuanto mayor es el tiempo de aprendizaje, menor es el riesgo de desempleo. Respecto al resto de variables consideradas, las recompensas del trabajo, medidas por la obtención de un ascenso, por el logro de un contrato indefinido y por la fecha de entrada en el empleo, constituyen factores que explican en gran medida el logro de la estabilidad laboral. En relación con la fecha de entrada en la ocupación, si el empleo se ha obtenido después de 1984, cuando se introducen las medidas de flexibilización del mercado de trabajo, se incrementa el riesgo de perderlo.

El tamaño dela empresa también influye en la estabilidad laboral. El riesgo de ir al paro es menor en las empresas de mayor tamaño. A este respecto, no hay que olvidar que en estas empresas suele ser característica la presencia de mercados internos de trabajo que ofrecen mayor seguridad en el empleo y mayores posibilidades de promoción. Si el empleo pertenece a las ramas de la Construcción y del M etal, que están caracterizadas por una alta rotación laboral, se incrementa el riesgo de ir al paro. Tal hecho también ocurre si el traba jo tiene lugar en las administraciones públicas; lo cual viene a señalar el alto grado de temporalidad que tienen actualmente los empleos ofrecidos por la Administración. Vivir en las Cuencas M ineras, que constituye una zona con graves problemas de empleo, se asocia con la pérdida de la ocupación. Finalmente, las mujeres tienen mayor riesgo de ir al paro que los varones.

Formación en el trabajo y movilidad directa entre empleos

En el análisis de las relaciones entre formación en el puesto y movilidad directa entre empleos, la variable dependiente es el número de meses que se permanece en un empleo antes de moverse hacia otro trabajo o de ser censurado. Las transiciones suponen el paso de un trabajo a otro sin que medie un período de paro, e implican un cambio de empleo y de empresa. El destino del cambio es la obtención, a juicio del encuestado, de un trabajo mejor ${ }^{7}$. Las variables incluidas en el modelo de C ox son las mismas que se han considera do en las transiciones de la ocupación al paro (tabla 6).

Tal como muestran los resultados, el tiempo de aprendizaje constituye un factor que desincentiva la movilidad de tipo voluntario, aquélla que responde a la iniciativa del propio trabajador. Si el trabajo requiere menos de un mes de aprendizaje, se incrementa notablemente el riesgo de que el trabajador busque otro empleo, mientras que si requiere más de tres meses tal riesgo disminuye.

En relación con el resto de factores que intervienen en este tipo de movilidad, la posesión de un contrato indefinido desincentiva igualmente la movilidad. Sin embargo, la obtención de un ascenso o la categoría ocupacional lograda

7. La movilidad hacia un trabajo mejor incluye los cambios de empleo que suponen el logro de una categoría ocupacional superior a la de origen, y los cambios hacia un empleo de la misma categoría ocupacional pero mejor recompensado. 
Tabla 6. M odelo Cox. M ovilidad hacia un trabajo mejor recompensado.

Variables

Tiempo de aprendizaje (Categoría residual: ninguno)

1. Menos de 1 mes

2. De 1 a 3 meses

3. De 3 a 12 meses

4. $M$ ás de 1 año

Tipo de vínculo laboral (Categoría residual: sin contrato)

1. Contrato indefinido

2. Contrato temporal

Rama de actividad (Categoría residual: servicios personales)

1. M inería, Energía y Q uímica

2. M etal

3. Resto industria

4. Construcción

5. Transporte y Comunicaciones

6. Comercio y H ostelería

7. Administraciones públicas

8. Enseñanza y Sanidad

9. $O$ tros servicios

$* * *$

$0,5865^{* * *}$

0,0053

$-0,4358 *$

$-0,4103^{*}$

$* * *$

$-1,0212 * * *$

$-0,0585$

**

$-0,3110$

$-0,1712$

$0,5076^{*}$

$-0,5112$

0,4792

$0,4303^{* *}$

$-0,5443$

$-0,8931 *$

$0,5863 * * *$

Nivel de estudios (Categoría residual: EGB)

1. Licenciatura

$* * *$

$1,2087 * * *$

2. Licenciatura incompleta

$-0,1852$

3. Diplomatura

0,4934

4. Diplomatura incompleta

$-0,2343$

5. BUP-COU

$-0,3426$

6. BUP-COU incompleto

$-0,3943 *$

7. FP2

$-0,0999$

8. FP2 incompleto

0,1042

9. FP1

0,2976

10. FP1 incompleto

Sexo (C ategoría residual: mujer)

№ de casos: 1.216 / Casos no censurados: 154/-2LL: 1774,614

$N$ iveles de significación: ${ }^{*} p<0,1000 * * p<0,0500 * * * p<0,0100$. 
no suponen un freno al cambio de empleo si dicho cambio trae consigo un trabajo mejor. Los trabajadores de las ramas de Comercio y H ostel ería, R esto de la industria y $O$ tros servicios (Seguros, Banca y Servicios a empresas) son los que experimentan una mayor movilidad laboral, al igual que los trabajadores con mayores recursos formativos: Ios licenciados universitarios. Por último, los hombres tienen mayor riesgo de cambiar de empleo que las mujeres. E ste resultado está en consonancia con las diferencias por género en los motivos aducidos para dejar el trabajo que vimos anteriormente.

\section{Formación en el trabajo y promoción laboral}

El último tipo de movilidad analizada es la que tiene lugar en el interior de las empresas, la llamada movilidad interna o promocional. El análisis de la influencia de la formación en el trabajo en este tipo de movilidad se ha efectuado mediante un modelo de regresión logística, en el que la variable dependiente adopta los valores de «1» si se ha producido un ascenso y «0» si la respuesta es no. El análisis está referido al último empleo dela historia laboral de los jóve nes, con independencia de si el joven está ocupado o no en el momento de la encuesta. En el modelo se ha incluido el tiempo de aprendizaje, junto con otras variables representativas de las características y los recursos personales, y de los puestos de trabajo y empresas. Como recursos y características personales se han considerado el nivel de estudios, el capital relacional, la antigüedad en el empleo y el género. Como características de los puestos y de las empresas se incluyen la categoría ocupacional, el tipo de vínculo laboral, la rama de actividad y el tamaño de la empresa (tabla 7).

Al igual que en las anteriores pautas de movilidad analizadas, la formación en el puesto de trabajo constituye un factor que interviene en los procesos de promoción dentro de las empresas. Esta relación se manifiesta especialmente en la asociación negativa que presentan los trabajos que requieren menos de un mes de formación con las promociones. Además de la formación en el puesto, en la probabilidad de obtener un ascenso intervienen de forma positiva la antigüedad en la empresa y la posesión de un contrato indefinido. El comportamiento de esta última variable parece indicar que el establecimiento de escal eras de promoción laboral es un mecanismo ligado al funcionamiento de los mercados internos de las em presas, caracterizados por relaciones de empleo a largo plazo. También, los trabajadores ocupados en empresas industriales privadas ascienden antes que los que trabajan en organizaciones públicas o en la Administración.

En cuanto a las características personales, las mujeres tienen menores probabilidades de ascender que los hombres. $D$ ado que la formación específica está teóricamente asociada con la presencia de mercados internos de trabajo, en los que es propio la existencia de escaleras de promoción laboral, cabría deducir, aunque sea de forma indirecta, que la discriminación en el acceso de este tipo de formación puede resultar del hecho que las mujeres tienen desiguales probabilidades de acceder a los puertos de entrada a tales mercados. 
Tabla 7. M odelo LO GIT. Probabilidad de ascenso en el último empleo.

Variables

$\mathrm{N}$ ivel de estudios (Categoría residual: EGB)

1. Licenciatura

***

2. Licenciatura incompleta

$-0,2509$

3. Diplomatura

0,2250

4. Diplomatura incompleta

$-1,9531 * * *$

5. BUP-COU

0,2582

6. BUP-COU incompleto

0,0350

7. FP2

$0,9197^{* * *}$

8. FP2 incompleto

0,3158

9. FP1

$-0,2622$

10. FP1 incompleto

Antigüedad en el empleo (Categoría residual: más de 3 años)

0,3253

0,6096

1. H asta 6 meses

$* * *$

2. D e 6 meses a 1 año

$-0,4662 *$

3. De 1 a 2 años

$-0,5707^{* *}$

4. De 2 a 3 años

0,0075

$0,4293 * *$

Tiempo de aprendizaje (Categoría residual: más de 1 año)

**

1. $\mathrm{N}$ inguno

$-0,7833^{* * *}$

2. M enos de 1 mes

$-0,1916$

3. De 1 a 3 meses

$0,3251 *$

4. De 3 a 12 meses

$0,3324 *$

Categoría ocupacional (Categoría residual: obreros no cualificados)

$* * *$

1. Profesionales y técnicos

0,3460

2. Administrativos y comerciales

0,1453

3. 0 breros cualificados

0,1633

Tipo de vínculo laboral (Categoría residual: sin contrato)

***

1. Contrato indefinido

$0,9532 * * *$

2. Contrato temporal

$-0,1355$

Rama de actividad (C ategoría residual: servicios personales)

**

1. M inería, Energía y Q uímica

$-0,2232$

2. M etal

0,5055

3. Resto de industria

$0,6537^{* *}$

4. Construcción

0,3465

5. T ransporte y Comunicaciones

0,4950

6. Comercio y H ostelería

0,1428

7. Administraciones públicas

$-0,7640 *$

8. Enseñanza y Sanidad

$-0,9242 * *$

9. 0 tros servicios

$-0,2362$

Sexo (Categoría residual: mujer)

$0,4436 *$

Constante

$-2,3977^{* * *}$

№ de casos: 1.185/-2LL: 754,120

$N$ iveles de significación: ${ }^{*} p<0,1000 * * p<0,0500 * * p<0,0100$. 
Por su parte, los recursos formativos representados por la educación formal no parecen influir en los sucesos promocionales, ya que únicamente los trabajadores con estudios de BUP incompletos presentan un coeficiente de signo positivo y significativo. En definitiva, se puede afirmar que la probabilidad que tiene un trabajador de ser ascendido depende en gran medida de factores estructurales, como puede ser su ubicación dentro de un mercado interno que ofrece estabilidad en el empleo y posibilidades de formación en el trabajo.

\section{Conclusiones}

Los análisis efectuados indican que la formación en el trabajo constituye un factor muy importante en la explicación de la estabilidad y el progreso laboral. Por ello resulta especialmente relevante determinar las características que se relacionan con la adquisición de una mayor formación. A este respecto, son Ios trabajadores con un contrato indefinido los que ocupan empleos que requieren un mayor tiempo de aprendizaje, así como aquélos cuyo empleo perte nece a la categoría de profesionales y técnicos. Los resultados también muestran la importancia que tiene para los empleos manuales industriales la adquisición de cualificaciones en el propio puesto de trabajo. Por el contrario, el tiempo de aprendizaje es sensiblemente menor en las mujeres que en los hombres, lo cual parece indicar que existe una cierta discriminación de mercado en el acceso a la formación. Las mujeres acceden a empl eos que ofrecen menos oportunidades formativas, con las consiguientes consecuencias negativas en sus posibilidades de carrera laboral.

El logro de estabilidad laboral depende mucho del acceso a un aprendiza je específico en los primeros empleos. Los trabajadores más estables son aquellos que recibieron más formación al entrar en la empresa, formación que suele denominarse de «acogida». Pero no sólo este tipo de formación protege frente al paro, sino que también se relaciona con una menor movilidad por parte del trabajador. Los trabajadores cuyos empleos requieren más formación tienen menos incentivos a cambiar el actual empleo por otro.

Además de reducir la rotación laboral, la formación en el trabajo constituye un factor que interviene de forma muy positiva en los procesos de promoción en el interior de las empresas. Este resultado sugiere que este tipo de formación está muy relacionada con la existencia de mercados internos de tra bajo; mercados caracterizados por el establecimiento de carreras de promoción internas y una mayor estabilidad laboral.

En general, los resultados obtenidos demuestran que la adquisición de cualificaciones en el trabajo es el factor que ejerce efectos más ben eficiosos en el inicio y la consolidación de una carrera profesional, por encima de la mera acumulación de experiencia laboral o de credenciales educativas. De ahí que sea especial mente importante el potenciar que las empresas ejerzan un mayor esfuerzo inversor en formación. En este punto hay que mencionar el papel que han tenido en el fenómeno que estamos analizando las políticas públicas en materia formativa y de empleo. En relación con las políticas formativas, el 
grave problema de desempleo ha llevado a los gobiernos ha centrar gran parte de su esfuerzo en programas de formación dirigidos a los desempleados. Sin embargo, esta situación está variando últimamente, y ya se observa un giro en la distribución de recursos públicos a nivel estatal y comunitario hacia la formación en la empresa (Sáez Fernández, 1997).

Por su parte, las políticas de empleo que se han llevado a cabo en España en los últimos años, con una legislación contractual que ha impulsado extraordinariamente la contratación temporal, han favorecido una rotación laboral que podríamos considerar artificial, superior a los ajustes de plantilla debidos a necesidades productivas y de mercado. Esta elevada rotación se ha manifestado especialmente en el proceso de inserción laboral juvenil; proceso que se ha caracterizado asimismo por el acceso al empleo a través de puestos de trabajo de bajo nivel de cualificación, para cuyo desempeño no se requiere prácticamente de un aprendizaje, y en los que la sustitución de un trabajador por otro no supone un grave coste para la empresa. En este aspecto, la situación puede variar si se consolidan políticas públicas tendentes a potenciar la contratación indefinida. En tal caso, se podrían originar cambios en las estrategias formativas y de gestión de recursos humanos de las empresas, orientados hacia un mayor uso y aprovechamiento de los mercados internos y del potencial formativo de los trabajadores.

\section{Anexo}

A continuación se detalla la definición operativa de las variables utilizadas en los diferentes análisis y los valores que adoptan las categorías de respuesta.

Tiempo de aprendizaje Variable que indica el tiempo de aprendizaje que el tra bajador considera necesario para desarrollar las tareas del puesto: «1» N inguno, «2» M enos de 1 mes, «3» D e 1 a 3 meses, «4» D e 3 meses a 1 año, «5» M ás de 1 año.

$\mathrm{N}$ ivel de estudios Variable tratada como categórica que indica el nivel de estudios al canzado en cada suceso o estado de la historia laboral, distinguiendo si es completado o no: «1» Licenciatura, «2» Licenciatura incompleta, «3» D iplomatura, «4» D iplomatura incompleta, «5»BU P-COU , «6»BU PCOU incompleto, «7»FP2, «8»FP2 incompleto, «9»FP1, «10»FP1 incompleto, «11»EGB.

Tipo de titulación. Variable que recoge las diferentes especialidades de FP y las carreras universitarias, junto con los estudios de BU P-COU y EG B: «1» FP1 Industria y C onstrucción, «2»FP1 Administrativa, «3»FP1 Servicios, «4»FP2 Industria y Construcción, «5»FP2 Administrativa, «6»FP2 Servicios, «7» D iplomaturas de Artey H umanidades, «8» D iplomaturas Científico-Tecnológicas, «9» D iplomaturas en CC. Sociales, «10»D iplomaturas Biosanitarias, «11» Licenciaturas de Arte y H umanidades, «12» Licencia- 
turas Científico-Tecnológicas, «13» Licenciaturas en CC. Sociales, «14» Licenciaturas Biosanitarias, «15»BU P-COU , «16» EGB.

Simultanei dad de estudios y trabajo. Variable que agrupa las diferentes situaciones de compatibilidad de estudios y trabajo en cada suceso de empleo de la historia laboral: «1»Trabaja y estudia FP, «2» Trabaja y estudia BU PCOU , «3»Trabaja y estudia una diplomatura, «4» Trabaja y estudia una licenciatura, «5» N 0 estudia.

C ategoría ocupacional. Variable que recoge las diferentes categorías ocupacionales de la población asalariada con los siguientes valores: «1» Profesionales y técnicos, «2»Administrativos y comerciales, «3» 0 breros cualificados, «4» O breros no cualificados.

Rama de actividad. Variable que indica la rama de actividad a la que pertenece el empleo. Sehan distinguido diez categorías: «1» M inería, Energía y Q uímica, «2»M etal, «3»R Resto de Industria, «4» Construcción, «5» Transporte y Comunicaciones, «6» Comercio y H ostelería, «7»Administraciones públicas, «8» Enseñanza y Sanidad, « $9 » 0$ tros servicios, «10» Servicios personales.

Tipo de contrato. Variable que indica el tipo de vínculo laboral del trabajador con la empresa: «1»C ontrato indefinido, «2»C ontrato temporal, «3» Sin contrato.

Tamaño de la empresa. Variable categórica que agrupa el tamaño de la empresa en los siguientes grupos: «1» H asta 10 empleados, «2» D e 11 a 50, «3» M ás de 50, «4» N S/N C. En relación con esta variable, el número de no respuestas que tuvo lugar durante la realización de la encuesta fue bastante elevado y por ello su uso sólo ha sido posible transformando la categoría de no respuesta en el valor residual de la variable.

Ascenso. Variable dicotómica que recogela mejora de categoría profesional en cada suceso de empleo: «1»H a obtenido un ascenso, «0» No lo ha obtenido.

Pruebas de sel ección para acceder al empleo. Variable que adopta las siguientes categorías de respuesta: «1» N o ha pasado por pruebas de selección, «2» U na oposición, «3» H a pasado por pruebas de selección.

C anal de acceso al empleo. Variable dicotómica que indica las fuentes de información por medio de las cuales se ha obtenido el empleo. Adopta los valores de «1» Si se ha obtenido por medio de terceras personas (capital relacional) y «0» Por otras vías.

Año de entrada en la ocupación. Variable dicotómica con valores «1» Entrada después de 1984, «0» Antes de 1984. 
Área de residencia. Adopta los siguientes valores: «1» Avilés, «2» Gijón, «3» Cuencas $M$ ineras, « $4 » 0$ viedo.

Tiempo de paro. M ide en meses el tiempo pasado en paro antes del suceso de empleo analizado: «1» N inguno, «2»D e 1 a 6 meses, «3» De 6 meses a 1 año, «4» D e 1 a 2 años, «5» M ás de 2 años.

Antigüedad en el empleo. Variable que mide en meses el tiempo pasado en cada empleo: «1» H asta 6 meses, «2» De 6 meses a 1 año, «3» D e 1 a 2 años, «4» D e 2 a 3 años, «5» M ás de 3 años.

Atracción por el empleo. Variable que recoge la atracción por diferentes elementos de empleo: «1»El salario, «2» Las tareas del puesto, «3» La seguridad en el empleo, «4» El prestigio de la empresa, «5» La confianza en el dueño, «6» Las condiciones de trabaj0, «7» La oportunidad de formarse, «8» Las oportunidades de promoción, «9» La oportunidad de empezar a trabajar, «10» 0 tros.

M otivos de la pérdida del empleo. Variable que indica las diferentes causas de pérdida del empleo: «1» Se cumplió el tiempo de contrato, «2»Encontrar otro empleo mejor, «3»D espido disciplinar o regulación de empleo, «4»A iniciativa del sujeto, «5» Por razones familiares.

Sexo. Variable dicotómica con valores de «1» Varón y «0» M ujer.

\section{Bibliografía}

Alba-Ram írez, A. (1996). «En busca del primer empleo: el precio de la experiencia». Economistas, núm. 70, p. 14-22.

Allison, E.; Allen, P. (1980). «M ale-Female Professionals: A M odel of Career Choices. Industrial Relations, vol. 19, núm. 3, p. 366-370.

AltonjI, J,G .; Spletzer, J.R. (1991). «Worker Characteristics, Job Characteristics, and the Receipt of on-the job Training». Industrial and Labor Relations Review, vol. 45, núm. 1, p. 58-79.

Arrow, K. J. (1991). «La educación superior como filtro». Economía y Sociedad, núm. 8, p. 143-161 (traducción en castellano de la versión inglesa «H igher Education as a Filter». Journal of Public Economics, 1973, vol. 2, p. 193-216.).

BARRON, J.M .; BISH OP, J.; D Un KELBERG, W.C. (1985). «Employer Search: The Interviewing and $\mathrm{H}$ iring of $\mathrm{N}$ ew Employees». The Review of Economic and Statistics, vol. LXVII, núm. 1, p. 43-52.

Barron, J.M.; BLACK, D .A.; LOEWENSTEIN, M .A. (1989). «ob M atching and Onthe J ob Training». En Journal of Labor Economics, vol. 7, núm. 1, p. 1-19.

BECKER, G. (1983). El Capital H umano. M adrid: Alianza Editorial (edición original, $2^{\mathrm{a}}$ ed., 1975).

Blossfeld, H . (1989). «U sing Cox M odels to Study M ultiepisode Processes». Socio logi cal M ethods \& Research, vol. 17, núm. 4, p. 432-448. 
Воотн, A. L. (1991). «ob-Related Formal Training: who receives it and what is it worth? Oxford Bulletin of Economics and Statistics, vol. 53, núm. 3, p. 281-293.

- (1993). «Private Sector Training and Graduate Earnings». The Review of Econo mics and Statistics, vol. LXXV, núm. 1, p. 164-170.

Cohen, Y.; Pfeffer, J. (1986). «O rganizational H iring Standards». Administrative Science Q uarterly, vol. 31, núm. 1, p. 1-24.

D o ERIN GER, P.B.; PIO RE, M .J . (1985). M ercados internos de trabajo y análisis laboral. M adrid: M inisterio de Trabajo (edición original de 1971).

FILER, R.K. (1986). «T he R ole of Personality and T astes in D etermining 0 ccupational Structure». Industrial and Labor Relations Review, vol. 39, núm. 3, p. 412-424.

García Blan co J.M.; Gutiérrez Palacios, R. (1996). La inserción laboral delos jóvenes, manuscrito. U niversidad de 0 viedo.

GaRCía ESPEjO, I. (1998). Recursos formativose inserción laboral dejóvenes. Colección M onografías núm. 158, M adrid: CIS-Siglo XXI.

GaRRID o Medin A, L.J. (1995). Diagnóstico sobreel paro juvenil y políticas para facili tar la entrada al primer trabajo. Colección «Estudios de Políticas», núm. 16. Gine bra: OIT.

GREEN, F. (1993). «T he D eterminants of Training of M ale and Female Employees in Britain». Oxford Bulletin of Economics and Statistics, vol. 55, núm. 1, p. 103-122.

Green halgh, C.; M AVRotas, G . (1994). «T he Role of Career Aspirations and Financial Constraints in Individual Acces to Vocational Training». O xford Economic Papers, vol. 46, núm. 4, p. 579-601.

G ReEN H ALGH , C.; SteWART, M . (1987). «T he Effects and D eterminants of Training».

Oxford Bulletin of Economics and Statistics, vol. 49, núm. 2, p. 171-190.

IbáÑ Ez PASCUAL, M . (1999). Género y familia en la inserción laboral. O viedo: Servicio de Publicaciones de la U niversidad de 0 viedo (en prensa).

KNIGHT, K.G.; LATREILLE, P.L. (1996). «Apprenticeship Training and D ay Release in UK Engineering: Some Cross-sectorial Evidence». British Journal of Industrial Rela tions, vol. 34, núm. 2, p. 307-314.

KN OKE, D.; KalleberG, A.L. (1994). «ob Training in U .S. Organizations». Ameri can Sociological Review, vol. 59, núm. 4, p. 537-546.

LYN CH , L.M . (1992). «Private-Sector T raining and the Earnings of Young W orkers». American Economic Review, vol. 82, núm. 1, p. 299-312.

M ARSDEN, D. (1994). M ercados detrabajo. Límites sociales de lasnuevas teorías. M adrid: M inisterio de Trabajo y Seguridad Social (edición original 1989).

O CDE (1994). Estudio de O CDE sobre el empleo: hechos, análisis estrategias. París.

PÉREZ IN FAN TE, J.I . (1988). «La política de empleo juvenil en España: regulación y resultados». Economía y Sociología del Trabajo, núm. 1-2, p. 91-100.

SÁez Fern Án D EZ, F. (1997). «Políticas de formación continua, balance y perspectivas». Revista del M inisterio de Trabajo y Asuntos Sociales, núm. 1, p. 79-87.

SAN D ELL, S.H .; SH APIRO, D . (1980). «W ork Expectations; H uman Capital Accumulation, and the Wages of Young W omen». The Journal of H uman Resources, vol.15, núm. 3, p. 335-352.

So Ren SEn, A.B.; Kalleberg, A.L. (1981). «An 0 utline of a Theory of the M atching of Persons to Jobs»En BERG, I (ed.). Sociological Perspectives on Labor M arkets, N ueva York: Academic Press, p. 49-74. 
So Ren SEn, A. B.; TUm A, N . B. (1981). «Labor M arket Structures and J ob M obility». Research in Social Stratification and M obility, vol. 1, p. 67-94.

SPENCE, M . (1991). «Indicadores del mercado de trabajo». Educación y Sociedad, núm. 8, p. 123-141 (traducción en castellano de la versión inglesa «ob M arket Signaling». Q uaterly Journal of Economics, 1973, vol. 87, núm. 3, p. 355-374).

ThuRow, L. (1975). Generating Inequality. N ueva York: Basic Books.

T U M AN . B. (1985). «Effects of Labor M arket Structure on Job Shift Patterns». En H eckman, J. . ; Burton, S. (eds.). L ongitudinal Analysis of Labor M arket D ata. Cambridge U niversity Press, p. 327-363. 